\title{
New immune therapy targets tumor-associated environment: from bone marrow to tumor site
}

\author{
Cellular \& Molecular Immunology (2012) 9, 1-2; doi:10.1038/cmi.2011.54; published online 12 December 2011
}

$\mathrm{T}$ umor-associated antigens (TAAs) and immune cells coexist in the tumor microenvironment. However, spontaneously developed cancer cells have coevolved with immune cells, and have utilized inflammatory signals and immune suppressive mechanisms to support and promote tumor progression. Subverting immune suppressive mechanisms and induction of potent and lasting anti-tumor immunity is the goal of cancer vaccination and immunotherapy. Given that there are vast numbers of genetic changes associated with carcinogenesis, it would not be unexpected that tumor cells express considerable neoantigens, and many cancers are immunogeneic. ${ }^{1,2}$ Nonetheless, spontaneous tumor eradication is rare. Although many tissue-specific proteins or tumor neoproteins are used as vaccines or antigens to prime and expand TAA-specific T cells, our knowledge of tumor rejecting antigens remains limited.

Active immunization and adoptive T-cell transfer therapy are the main strategies used thus far for cancer immunotherapy. These strategies are designed to overcome the deficiency in TAA-specific T-cell priming and the reduced T-cell effector function in patients with cancer. Cancer vaccines rely on immunization of patients with antigenic peptides, proteins or DNA expressed by tumor cells or dendritic cells or viral vectors. However, it is well known that tumor vaccination has shown limited clinical success. ${ }^{3}$ This is consistent with the finding in murine models that the presence of large numbers of TAA-specific T cells is insufficient to mediate tumor regression. ${ }^{2,4}$ Adoptive T-cell transfer therapies, in which $\mathrm{T}$ cells are isolated from the tumor or peripheral blood and expanded in vitro in an antigen-specific manner, have shown promise in selective patients with melanoma. ${ }^{5}$ However, the requirement for the knowledge of the tumor rejection antigens and the inability to have sufficient quality $\mathrm{T}$ cells from tumor tissues currently limit its application.

In this special issue, we have discussed two important strategies for generating potent and lasting anti-tumor immunity. The first strategy is to subvert immune suppressive networks in the tumor microenvironment. ${ }^{6-8}$ Regulatory $\mathrm{T}$ cells (Tregs) are one of the most important immunosuppressive components. ${ }^{8,9}$ While most studies focus on Tregs in tumor tissues or draining lymph nodes, here we emphasized that bone marrow is a reservoir for activated Tregs, and suggested how to target molecules important for Treg expansion and trafficking in the bone marrow in patients with cancer. The second strategy is to optimize conventional and anti-biological modalities to directly target tumor and adjacent tumor tissue, and mobilize and

Correspondence: Dr Y-X Fu, Department of Pathology and Committee on Immunology, University of Chicago, Chicago, IL 60637, USA.

E-mail: yfu@uchicago.edu

Received 9 November 2011; accepted 10 November 2011 expand anti-tumor immunity in the tumor microenvironment which results in tumor eradication.

Why do we need to focus on bone marrow in patients with cancer? Bone marrow is a predetermined metastatic location for multiple human tumors. In addition to its unique biological elements, in this special review issue, Zhao et al review the role of BM for tumor growth and immune suppression-there has been a growing realization that the unique immune microenvironment plays an important role in tumor growth and metastasis in bone marrow. Understanding this unique immune microenvironment and the underlying cellular and molecular mechanisms will definitely generate novel insight into cancer therapy and particularly cancer bone metastasis. Several immune suppressive elements, including Tregs, form immunosuppressive networks in the tumor microenvironment. ${ }^{9-11}$ It appears that this applies to bone marrow and that bone marrow is an immune suppressive environment. The levels of Tregs are significantly higher in bone marrow as compared to other organ compartments. In patients with prostate cancer, the numbers of Tregs are further increased in bone marrow. This is attributed to active Treg recruitment and expansion in bone marrow of prostate cancer patients with bone metastasis. Treg bone marrow trafficking is largely mediated by CXCR4/ CXCL12 signaling pathway. Activated Tregs express functional CXCR4 and efficiently migrate towards CXCL12. High levels of CXCL12 are found in bone marrow. CXCL12 is derived from bone marrow stromal cells and tumor cells including prostate cancer. ${ }^{12}$ Treg expansion in bone marrow is mediated by RANK/RANKL. In patients with prostate cancer, Tregs express high levels of RANKL, and dendritic cells express high levels of RANK. ${ }^{13}$ Bone marrow Tregs tilt the balance between osteoclast and osteoblast activity, which may contribute to osteoblastic bone lesions that characterize prostate cancer. Therefore, CXCR4/CXCL12 and RANK/RANKL are crucial molecular signaling pathways for Treg bone marrow trafficking and expansion, respectively. ${ }^{13}$ Thus, we suggest that the combinatorial blockade of these two signaling pathways would be a valid option to treat cancer patients with bone metastasis including prostate cancer and breast cancer.

How do we directly target tumor and adjacent tumor tissue? It is well known that conventional tumor therapy (e.g., chemotherapy, radiotherapy) and biological antibody therapy (e.g., anti-Her2/neu) directly target tumor cells and mediate tumor killing. In this issue, Yang et al. summarize recent progresses in understanding the potential new immune mechanism for conventional treatment mediated tumor regression. Compelling evidence suggests that therapeutic 
efficacy of many cancer treatments depends on host immune responses: (i) antibodies to oncogenic receptors are thought to induce apoptosis of tumor cells directly or indirectly via FcR. However, antibody-mediated tumor regression depends on $\mathrm{T}$ cells and additional immunotherapy can greatly enhance antibody-initiated immunity. ${ }^{14,15}$ Furthermore, kinase inhibitors can preferentially target tumor tissues and induce tumor regression partially depending on $\mathrm{T}$ cells; ${ }^{16,17}$ (ii) the current mechanistic explanation for the clinical efficacy of local ablative radiation therapy (RT) centers on the induction of lethal DNA damage in tumor cells or tumor-associated stroma. Surprisingly, ablative localized RT on a tumor largely depends on T cells. ${ }^{18}$ RT-induced danger signal molecules such as HMGB1 to active dendritic cell through TLR4/MyD88 signal pathway are essential for the therapeutic effect of RT; ${ }^{19}$ (iii) chemotherapy was designed to kill fast dividing tumor cells by targeting essential replicative steps, such as DNA replication and transcription. This therapy has been very successful in reducing tumor burden and is the standard treatment for many types of cancer. However, the therapeutic effect of chemotherapy is also dependent on host immune responses. ${ }^{19}$ It is conceivable that some chemotherapies can trigger danger signals to bridge innate and adaptive immunity. Therefore, future studies examining how different cytotoxic drugs affect the immune system are necessary. In addition, understanding the proper timing and dosing of chemotherapy for combination with immunotherapy will be important for inducing synergy between these therapies, which results in better antitumor activity; and (iv) targeted immunotherapy to primary tumor tissues can generate immunity against distal tumor tissues. Based on the lessons learned from autoimmunity, tertiary lymphoid structure is essential and sufficient to induce destruction. ${ }^{20}$ We suggest that the generation of tertiary lymphoid structure inside tumor tissues will change the local microenvironment and result in tumor eradication. In support of this possibility, targeting tumors with the tumor-necrosis factor superfamily member (TNFSF14 or LIGHT) creates tertiary lymphoid structure, brings immune responses inside tumor tissues and generates more cytotoxic $\mathrm{T}$ cells to control local tumor and distal tumors. ${ }^{21}$

In summary, current cancer treatment by removing primary tumor immediate after diagnosis might leave not enough tumor antigens in situ for subsequent immunotherapy. Instead, we suggest that targeting primary tumor as the site of TAA-specific priming prior to surgical resection may offer a great advantage to induce potent and lasting anti-tumor immunity that is sufficient to eradicate dormant metastatic tumors. Future anti-tumor therapy requires redesigning conventional treatment and target and utilizes tumor tissues with immunotherapy to achieve the most efficient therapeutic efficacy, and ultimately results in the control of metastatic disease and tumor relapse.
Yang-Xin $\mathrm{Fu}$

Department of Pathology and Committee on Immunology, University of ChicagoChicago, IL, USA

1 Boon T, van der Bruggen $\mathrm{P}$. Human tumor antigens recognized by T lymphocytes. J Exp Med 1996; 183: 725-729.

2 Yu P, Rowley DA, Fu YX, Schreiber $\mathrm{H}$. The role of stroma in immune recognition and destruction of well-established solid tumors. Curr Opin Immunol 2006; 18: 226-231.

3 Rosenberg SA, Yang JC, Restifo NP. Cancer immunotherapy: moving beyond current vaccines. Nat Med 2004; 10: 909-915.

4 Overwijk WW, Theoret MR, Finkelstein SE, Surman DR, de Jong LA, Vyth-Dreese FA et al. Tumor regression and autoimmunity after reversal of a functionally tolerant state of self-reactive CD8 ${ }^{+}$T cells. J Exp Med 2003; 198: 569-580.

5 Dudley ME, Wunderlich JR, Robbins PF, Yang JC, Hwu P, Schwartzentruber DJ et al. Cancer regression and autoimmunity in patients after clonal repopulation with antitumor lymphocytes. Science 2003; 298: 850-854.

6 Zhou P, Fang X, McNally BA, Yu P, Zhu M, Fu YX et al. Targeting lymphotoxinmediated negative selection to prevent prostate cancer in mice with genetic predisposition. Proc Natl Acad Sci USA 2009; 106: 17134-17149.

7 Pardoll DM. Spinning molecular immunology into successful immunotherapy. Nat Rev Immunol 2002; 2: 227-238.

8 Zou W. Immunosuppressive networks in the tumour environment and their therapeutic relevance. Nat Rev Cancer 2005; 5: 263-274.

9 Zou W. Regulatory T cells, tumour immunity and immunotherapy. Nat Rev Immunol 2006; 6: 295-307.

10 Curiel TJ, Coukos G, Zou L, Alvarez X, Cheng P, Mottram P et al. Specific recruitment of regulatory $T$ cells in ovarian carcinoma fosters immune privilege and predicts reduced survival. Nat Med 2004; 10: 942-949.

11 Yu P, Lee Y, Liu W, Krausz T, Chong A, Schreiber H et al. Intratumor depletion of CD4 ${ }^{+}$ cells unmasks tumor immunogenicity leading to the rejection of late-stage tumors. J Exp Med 2005; 201: 779-791.

12 Zou L, Barnett B, Safah H, Larussa VF, Evdemon-Hogan M, Mottram P et al. Bone marrow is a reservoir for $\mathrm{CD} 4^{+} \mathrm{CD} 25^{+}$regulatory $\mathrm{T}$ cells that traffic through $\mathrm{CXCL12/}$ CXCR4 signals. Cancer Res 2004; 64: 8451-8455.

13 Zhao E, Wang L, Dai J, Kryczek I, Wei S, Vatan L et al. Regulatory T cells in the bone marrow microenvironment in patients with prostate cancer. Oncolmmunology 2011; in press.

14 Park S, Jiang Z, Mortenson ED, Deng L, Radkevich-Brown O, Yang X et al. The therapeutic effect of anti-HER2/neu antibody depends on both innate and adaptive immunity. Cancer Cell 2010; 18: 160-170.

15 Stagg J, Loi S, Divisekera U, Ngiow SF, Duret H, Yagita H et al. Anti-ErbB-2 mAb therapy requires type I and II interferons and synergizes with anti-PD-1 or anti-CD137 mAb therapy. Proc Natl Acad Sci USA 2011; 108: 7142-7147.

16 Balachandran VP, Cavnar MJ, Zeng S, Bamboat ZM, Ocuin LM, Obaid H et al. Imatinib potentiates antitumor $\mathrm{T}$ cell responses in gastrointestinal stromal tumor through the inhibition of Ido. Nat Med 2011; 17: 1094-1100.

17 Rakhra K, Bachireddy P, Zabuawala T, Zeiser R, Xu L, Kopelman A et al. CD4 ${ }^{+}$T cells contribute to the remodeling of the microenvironment required for sustained tumor regression upon oncogene inactivation. Cancer Cell 2010; 18: 485-498.

18 Lee Y, Auh SL, Wang Y, Burnette B, Meng Y, Beckett M et al. Therapeutic effects of ablative radiation on local tumor require $\mathrm{CD} 8^{+} \mathrm{T}$ cells: changing strategies for cancer treatment. Blood 2009; 114: 589-595.

19 Apetoh L, Ghiringhelli F, Tesniere A, Obeid M, Ortiz C, Criollo A et al. Toll-like receptor 4-dependent contribution of the immune system to anticancer chemotherapy and radiotherapy. Nat Med 2007; 13: 1050-1059.

20 Lee Y, Chin RK, Christiansen P, Sun Y, Tumanov AV, Wang J et al. Recruitment and activation of naive $T$ cells in the islets by lymphotoxin beta receptor-dependent tertiary lymphoid structure. Immunity 2006; 25: 499-509.

21 Yu P, Lee Y, Liu W, Chin RK, Wang J, Wang Y, Schietinger A et al. Priming of naive T cells inside tumors leads to eradication of established tumors. Nat Immuno/ 2004; 5: 141-149. 